Provided for non-commercial research and education use. Not for reproduction, distribution or commercial use.

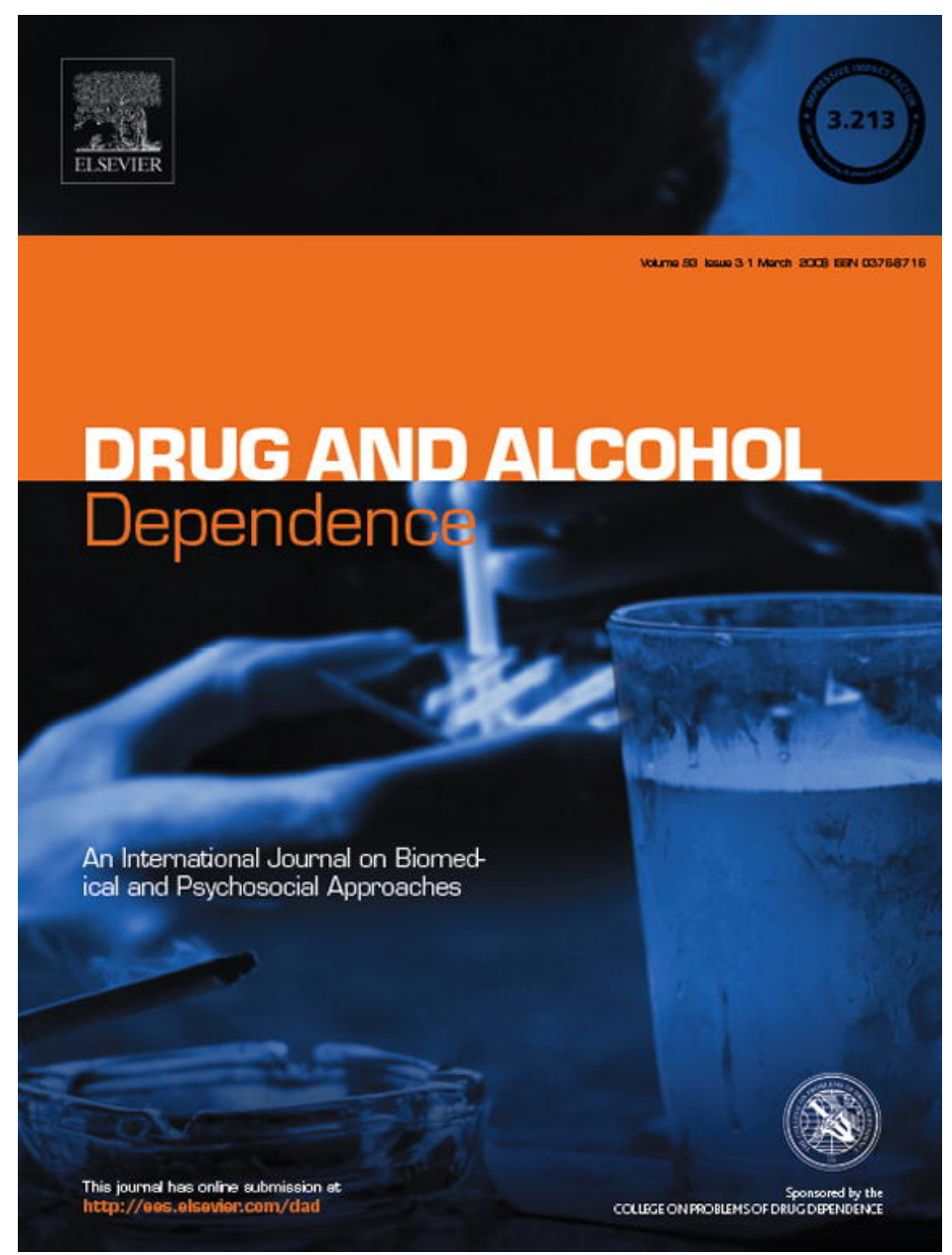

This article was published in an Elsevier journal. The attached copy

is furnished to the author for non-commercial research and education use, including for instruction at the author's institution, sharing with colleagues and providing to institution administration.

Other uses, including reproduction and distribution, or selling or licensing copies, or posting to personal, institutional or third party websites are prohibited.

In most cases authors are permitted to post their version of the article (e.g. in Word or Tex form) to their personal website or institutional repository. Authors requiring further information regarding Elsevier's archiving and manuscript policies are encouraged to visit: 


\title{
Commentary
}

\section{Researching the intoxicated: Informed consent implications for alcohol and drug research}

\author{
Judith Aldridge $^{\mathrm{a}, *}$, Vikki Charles ${ }^{\mathrm{b}}$

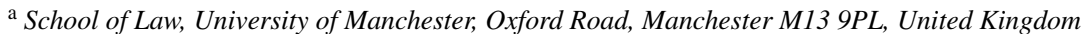 \\ ${ }^{\mathrm{b}}$ National Addiction Centre, Institute of Psychiatry, Kings College London, \\ 4 Windsor Walk, Denmark Hill, London SE5 8AF, United Kingdom
}

Received 3 April 2007; received in revised form 4 September 2007; accepted 5 September 2007

Available online 22 October 2007

\begin{abstract}
This article considers the informed consent process in relation to carrying out research with intoxicated participants in 'field' research settings. There is little discussion in the literature of the potential problems that the intoxication of research participants may pose to research. Intoxication is a potential problem for all researchers but is heightened in field research that takes place in settings where participants are likely to be intoxicated, such as licensed venues, in drug consumption rooms, or police custody suites. The risks to research participants that intoxication poses should not be resolved by electing not to do research with intoxicated participants; it is argued that these risks can be managed to some extent, and are offset by the benefits of such research. Moreover, intoxication (and the impairment of cognitive functions relevant to valid informed consent) may not always be identifiable through behavioural or biochemical methods of detection. The search for accurate and field-practical methods for identifying intoxication amongst participants is useful, but not the only strategy for researchers who want to ensure the validity of the consent process. Suggestions are provided for devising research protocols that acknowledge and accept intoxication of research participants and attempt to protect them. One solution is to side-step identification of intoxication per se as a strategic objective in the consent process, and turn instead to established methods for ensuring that information has been understood by potential research participants.
\end{abstract}

(C) 2007 Elsevier Ireland Ltd. All rights reserved.

Keywords: Intoxication; Ethics; Informed consent; Research process; Alcohol; Illegal drugs

\section{Introduction}

Intoxication by alcohol and other psychoactive illegal/illicit drugs $^{1}$ may compromise the informed consent process in research. Intoxication can impair cognition and judgement that are essential in order that potential research participants can understand what participation in research involves, and thus make informed decisions about their participation.

Researchers may be more likely to encounter intoxicated research participants when the populations from which researchers draw their samples are drug and alcohol users themselves, and in settings where participants are likely to

\footnotetext{
* Corresponding author.

E-mail addresses: Judith.Aldridge@manchester.ac.uk (J. Aldridge), Vikki.Charles@iop.kcl.ac.uk (V. Charles).

${ }^{1}$ Under consideration here are the psychoactive substances primarily associated with intoxication in field-based studies: alcohol, and primarily illegal/illicit drugs such as cannabis, ecstasy, and heroin.
}

be intoxicated at the time of obtaining consent: in the homes of research participants, in 'the streets', in drug consumption rooms, in licensed and other social venues. For example, the last 15 years have seen a resurgence of venue-based drug and alcohol research with the study of raves and club drug use, from the first 'dance drug' research carried out and published in the early 1990s in Britain (Newcombe, 1992) followed by studies in Britain (Deehan and Saville, 2003; Measham et al., 2001; Release, 1997; Riley et al., 2001; Sanders, 2005; Silverstone, 2006), the US (Kelly, 2006; Perrone, 2006; Yacoubian et al., 2003), Australia (Gourley, 2004), and elsewhere in Europe (Tossmann et al., 2001; van de Wijngaart et al., 1999) employing this venue-based design. Quantitative in situ research has also involved data collection with intoxicated participants, in which consent will, of necessity, have been obtained from intoxicated participants. Recently for example, Voas et al. (2006) carried out surveys along with alcohol breath tests and oral fluid drug assays using their 'portal' method outside electronic music dance events to estimate levels of drug and alcohol consumption. 
We discuss here research participant intoxication regarding the informed consent process where research occurs 'in the field', as opposed to in other more controlled settings.

\section{How researchers have dealt with intoxicated research participants}

The alcohol and illegal drug research literature provides scant discussion or guidance in relation to carrying out research with intoxicated participants. This suggests that many researchers who encounter intoxication amongst their research participants particularly in field research where intoxication may be prevalent - implicitly accept that research participants may be intoxicated without questioning and unpacking issues around that intoxication. Researchers who have acknowledged the intoxication of their research participants have dealt with it in one of two ways: (1) excluding intoxicated participants; or (2) excluding those deemed 'too intoxicated' to participate.

The first method is the more conservative: excluding the intoxicated from (at least) the consent process, and often from the research as well. A US survey found that even amongst researchers conducting primarily (non-field-based) clinical outcome studies, few had a policy for dealing with intoxicated participants, and of those who did, 'most indicated they would attempt to keep the participant at the site until sober or until transportation was arranged' (McCrady and Bux, 1999 p. 190). Others, in discussing research that involves administering the 'substance of dependence' to a drug user (College of Problems of Drug Dependence, 1995; Foddy and Savulescu, 2006), argue that heroin users are sufficiently free to consent to receive their drug of addiction, but that consent must occur when the addict is sober. The underlying assumption here is that the consent process - or even the research itself - may be compromised by the intoxication of research participants.

The second method for dealing with intoxication involves 'screening out' the more extremely intoxicated from being selected for research, and is less conservative because only intoxication beyond a particular level is deemed problematic. Deehan and Saville assessed visible signs of intoxication in their research with club-goers in six venues in England on a scale from 0 'no intoxication' to 5 'extremely intoxicated'. Only those $90 \%$ of potential interviewees considered 'able to take part in the interview comfortably' were approached (scoring from 0 to 2 on the scale) (2003). Those researching drug use by detainees in Australia (Mouzos et al., 2006), and Britain via NEW-ADAM (Bennett, 2000) have also excluded from their research those deemed 'too intoxicated' to interview. The British NEW-ADAM detainee study assessed 'fitness for interview' in part on the basis of drug and/or alcohol intoxication, and those deemed unfit included 29\% for alcohol and 2\% for other drugs, on the basis of an initial impressionistic subjective assessment by the custody sergeant. However, as Bennett points out, this did not guarantee that interviewees were not intoxicated at the time of interview, as it was observed that some of them found it hard to keep awake (a feature of substantial use of depressant drugs such as alcohol or heroin) (Bennett, personal communication). This method may have been useful for 'skimming off' the extremely intoxicated, but clearly was not able to identify all research participants who were intoxicated in ways that interfered with data collection.

Both approaches address and problematise research participant intoxication. Albeit in different ways, each approach involves assessing intoxication in order to identify and exclude intoxicated potential participants. We turn now to problems in assessing intoxication.

\section{Assessing intoxication}

Intoxication involves being under the influence of the effects of one or more psychoactive substances that may alter emotional state, perception, judgement and performance. However, intoxication is not a straightforwardly identifiable state that occurs in an easily measurable way when a psychoactive substance is ingested, regardless of place, expectation, and individual differences. Although biochemical markers for the presence of a substance have sometimes been demonstrated to correlate with the subjective effects of intoxication (e.g., Cone and Huestis, 1993), biochemical markers cannot accurately gauge intoxication itself. In relation to alcohol, for example, blood alcohol concentration (BAC) is not a reliable indicator of intoxication since the same BAC may have different effects in different people, and different effects in the same person across different circumstances including physical condition, personality, mood and situation (Krober, 1998). Regarding the substances that field researchers typically encounter - alcohol and illicit drugs biochemical detection is not suited to identifying intoxication because it is geared to identifying the presence of substances or their metabolites in the body. As such, biochemical detection does not take into account tolerance, rate of substance metabolism, and other individual and context differences.

Some have turned to behavioural approaches to identifying impairment thought to result from intoxication (e.g., Burns and Moskowitz, 1977) because of (1) the inability of biochemical detection to serve as a measure of intoxication; and (2) the need for field tests that do not involve expensive, bulky equipment or specialised technical expertise. However, success with the use of behavioural approaches to detecting impairment due to intoxication with illicit drugs is mixed, having been used successfully with some substances but not others. For example, Papafotiou et al. (2005) found that impaired performance on field tests assessing driving impairment was positively correlated with THC dose; however, field test results were not correlated with low levels of amphetamine intoxication (Silber et al., 2005). Recent evidence by Perham et al. (2007) suggests that subjective ratings of the physical manifestations of drunkenness (staggering gait, slurred speech, glazed eyes) of city centre drinkers by trained observers corresponded with blood alcohol concentrations. However, Brick and Carpenter (2001) found that police officers, watching a video-recorded interview, were able to correctly identify that alcohol-intoxicated subjects had been drinking only at relatively high levels of blood alcohol concentration (BAC 0.15-0.16\%). Thus, intoxication may be difficult to gauge even for police officers who are likely to be both trained in doing so, and who encounter alcohol intoxication frequently. Overall, these results suggest that observed behaviour is often 
not a very accurate predictor of substance level, suggesting that it might not always be a useful tool for researchers concerned about ability to give informed consent. Moreover, it is likely that intoxication levels that affect cognitive functions, thus in turn affecting the consent process, may not always be associated with observable impairment. Again therefore, behavioural approaches to identifying impairment will have only limited utility for researchers concerned about the ability of participants to give informed consent.

\section{The impact of intoxication on informed consent}

Because of the effect of intoxication by some psychoactive substances on cognition and judgement, it is likely that intoxication could impact a participant's capacity to understand explanations about research and on the capacity to make decisions about participation, by for example, impairing judgements about the merits or drawbacks of participation. It is likely that the impact of intoxication by some psychoactive drugs on feelings or emotional state (Parker et al., 1998) (e.g., happy, sad, scared, vulnerable and empathetic) may also colour perceptions and judgements in such a way as to influence decision making. There is research to suggest that alcohol intoxication affects suggestibility (Santtila et al., 1999), risk-taking (Lane et al., 2004) and moral reasoning (Fincham and Barling, 1979), all variables that could play a role in informed consent. Intoxication may therefore increase the chances of uninformed consent, reluctant consent, or consent that is later regretted.

There has been no systematic research on the impact of intoxication on the informed consent process. However, there is some evidence that intoxication may increase willingness to consent to research, and thus influence decision making. In venue-based research on dance drug use by Measham and colleagues, fieldwork staff regularly reported their perceptions that intoxication affected willingness to consent to interview. Indeed, respondents agreeing to an interview reported having already consumed more alcohol (8.3 units) than those who refused (6.6 units), a statistically significant difference, suggesting that alcohol intoxication may increase willingness to participate (Measham et al., 2001, p. 89).

\section{Ethical principles to guide research with intoxicated participants}

Ethical decision making in most medical, health-related and social research draws on principles which are relevant to conducting research with intoxicated participants: (1) autonomy: participants should be free to participate; (2) non-malificience: participants should be protected from harm; (3) beneficience: the benefits of research should outweigh the risks; (4) justice: people should be treated equally (Beauchamp and Childress, 2001).

Intoxication could be seen to compromise the autonomy of would-be research participants to consent, thus potentially leading to harm. On the other hand, the benefits of increased understanding generated about alcohol and other drug users in natural settings where intoxication is prevalent could be seen to outweigh the risks. This kind of research has a long and distinguished history, much of it influenced by the 'Chicago School' style of urban ethnography: Dai's Opium Addiction in Chicago (1937, 1970); Lindesmith's Opiate Addiction (1947, 1968); and Preble and Casey's Taking Care of Business (1969). Although research with some groups (e.g., the very young or old, those with physical or mental disabilities) can present special challenges in order for researchers to achieve their inclusion in research, it has been argued that to exclude them from research conflicts with the principle of justice through discrimination (Schuklenk, 2000) or through an unwillingness of researchers to speak directly to respondent groups that present challenges (Rodgers, 1999). Similar thinking can be applied to research with the intoxicated.

Some researchers have addressed consent issues for carrying out research with such 'vulnerable' groups where cognition and judgement may be impaired or deficient in such a way that normal procedures for obtaining informed consent are inappropriate or insufficient. The analogy with intoxication is not perfect. An intoxicated person, qua their intoxication, is not inevitably 'vulnerable'; however, it is their status as potentially vulnerable that concerns us here. Moreover, insights into the informed consent process particularly from those researching people with mental disabilities will be limited to some extent, since the incapacities addressed for that population group will be relatively enduring, whereas the relevant incapacities for intoxicated people will be relatively transitory. Recommendations that flow from these and other insights are considered below.

\section{Recommendations}

Although some researchers have taken a conservative stance on the problem of intoxication to the informed consent process by determining to exclude intoxicated participants from aspects of the research process (i.e., considering the risks to the participant too great), we contend here that the problem that intoxication poses should not be resolved by electing not to do research with intoxicated participants: (1) it is not possible completely to avoid the intoxicated, given the prevalence of the use of and intoxication by alcohol and illicit drugs; (2) intoxication is just one of a number of 'altered states' in which individuals find themselves, that include other common states such as stress and heightened emotions. Attempting to secure the participation of research participants who are in a 'pure' state (free from intoxication or other factors that may interfere with their cognitive processes in a way that impacts on providing consent) is likely to prove impossible; (3) both biochemical and behavioural methods for identifying intoxication (with a view to excluding the intoxicated) are problematic. We should rather seek to devise research protocols that acknowledge intoxication and protect research participants, thus off-setting risk in the form of potential harm to participants.

\subsection{Ensuring understanding}

One suggestion to enable people of varying capacities to consent to participate in research is to ensure that information 
provided to them is comprehensible and appropriate, and then check that the information has actually been understood. Sudore et al. (2006) have demonstrated a method of 'teaching then testing' potential respondents on information about their study, with a view to excluding from the consent process those who, after repeated teaching/testing, are unable to demonstrate adequate understanding. Regarding intoxicated participants for whom cognitive functioning may be diminished or altered, researchers need to have appropriate expectations regarding a participant's ability to take in, process and act on the information they have been given. Researchers may therefore consider building extra time into research protocols to check that understanding has occurred. For field research (which often takes place in busy or chaotic environments), a verbal variant of this approach could be used. The advantage of this approach is that it sidesteps the problem of assessing intoxication per se, and instead jumps directly to attempting to ensure that information about the research has been understood in spite of intoxication. As has been argued, in relation to carrying out research with people with mental disabilities (Fisher, 2003), that enhancing aspects of the consent setting that reduce consent vulnerability (i.e., providing comprehensible information and ensuring comprehension) may be preferable to conceiving consent competence as a 'state' within an individual; this may be a particularly important insight as regards intoxication, which is mostly a relatively transitory and changeable state.

\subsection{Extending the timeframe for consent and consent withdrawal}

Lawton (2001), commenting on her ethnographic research in a hospice, found that changes in the condition of patients meant that they were not always able to state whether they still wanted to take part in her research. The similarity between intoxicated participants and Lawton's dying patients lies in the fact of their conditions being changeable in ways that may be important regarding ongoing consent to participate. It is likely that levels of intoxication will vary over fairly short spaces of time (certainly hours, but even over shorter periods than that). Given the connection between intoxication and emotions/feelings and various cognitive processes, the willingness of participants to continue may, during the course of participation, change. If consent to participate in research should be treated as a process rather than as a one-off event, as has been argued (e.g., Ramcharan and Cutcliffe, 2001), it is sensible for fieldworkers to be alert - and remain alert - to changing signs of unwillingness to participate and to remind research participants where appropriate that they may terminate their participation at any time. Rodgers (1999) argues that there are signs that participants use aside from verbal dissent to signify a wish to opt out of participation that has already begun. A participant who becomes distracted/disinterested/agitated/irritated during an interview could be reluctant to continue. Being alert to these signs, checking that participants are happy to continue, and re-emphasising their right to withdraw at any time, should be built into research protocols and fieldworker/interviewer training.
Research participants may have regrets about their participation in the minutes, days or weeks following their participation. A standard de-brief should allow participants the space to reconsider their participation before fieldworker and participant have broken contact. The fieldworker can provide contact details that participants can easily keep or take away with them in a small and portable 'credit card' size. This allows the possibility for a former participant to initiate contact so that consent can be retroactively withdrawn, and effectively extends the time frame over which the process of providing consent can occur into periods in which former subjects are sober. The idea of retroactive withdrawal of consent is controversial, not least because it raises the rarely articulated issue of ownership of data; in other words, what are the limits of the 'rights' of a former research participant to control the data that resulted from their participation (see Wiles et al., 2006)? There are complexities in relation to retroactive withdrawal of consent that researchers will need to anticipate in their research protocols: what time limit for withdrawal is suitable, and how data already collected will be handled.

When researchers know that candidates for participation in research are under the influence of alcohol or illegal drugs, they may ask those who have already indicated a willingness to participate whether they believe that the effects of their consumption may have influenced their willingness. This question could provide potential respondents with the specific opportunity to reflect on their intoxication in relation to their willingness to participate in the research.

\subsection{Training fieldwork staff for intoxication awareness}

Research should be carried out by staff trained to be sensitive to signs of intoxication outside of the more expected and common alcohol intoxication (loss of co-ordination, staggering gait, drowsiness, slurred speech and glazed eyes). Signs of intoxication associated with other illicit drugs may also be assessed (paranoia, anxiety, eye-rolling, pupil dilation/constriction, head movements or jerks). It may be neither feasible nor desirable that research staff should be clinically trained, but they should have knowledge about the typical effects of the substances they are likely to encounter during fieldwork, and be trained to be aware of these, remembering to remind participants of their right to withdraw, particularly when observable signs of intoxication appear to change.

\subsection{Excluding the obviously intoxicated at the outset}

In spite of the limitations of visual/behavioural approaches to identifying intoxication, the use of an initial visual assessment of intoxication in order to screen out the most obviously and extremely intoxicated research participants may nevertheless be good practice for researchers (e.g, Bennett, 2000; Deehan and Saville, 2003). Although some very intoxicated participants may 'slip through the net' (Bennett, personal communication), excluding at least the most obviously intoxicated is a feasible and achievable objective. 


\section{Further research}

Although this contribution addressed the implications for the informed consent process of research participant intoxication where research occurs 'in the field', as opposed to in other more controlled settings, the implications apply also to some extent to research carried out in more controlled (for example clinical) settings, and also where research is conducted in the general population, such as in household surveys. The prevalence of drug and alcohol use is well documented; the prevalence of intoxication in the general population with the range of psychoactive substances remains undocumented. For example, it may be useful to for household survey researchers to know how often people are likely to be intoxicated in their homes during data collection.

Indeed, intoxication as a phenomenon remains underanalysed within social research approaches to drugs and alcohol research. In order to address this, social researchers need to develop methods of conceptualising, operationalising and measuring, and explaining and theorising intoxication for a range of substances. Of particular importance should be assessing the effect of intoxication on validity and accuracy of self-reports. More systematic research is required to establish the effects of intoxication by different drugs, or combinations of drugs, on the consent process across diverse research contexts.

\section{Conflict of interest}

None.

\section{Acknowledgements}

Many thanks to Trevor Bennett, Karen Clarke, Phil Edwards, Angela Melville, Toby Seddon and anonymous reviewers for their comments and suggestions.

Work in the preparation of the manuscript was supported by internal funds from the institutions of each author.

Contributors: Vikki Charles conducted some of the informed consent literature search and summarised this literature. Judith Aldridge drafted the manuscript. Both authors have contributed to and approved the final manuscript.

\section{References}

Beauchamp, T.L., Childress, J.F., 2001. Principles of Biomedical Ethics. Oxford University Press, Oxford.

Bennett, T., 2000. Drugs and Crime: The Results of the Second Developmental Stage of the NEW-ADAM Programme. Home Office Research Study 205, London. http://www.homeoffice.gov.uk/rds/pdfs/hors205.pdf.

Brick, J., Carpenter, J.A., 2001. The identification of alcohol intoxication by police. Alcohol. Clin. Exp. Res. 25, 850-855.

Burns, M., Moskowitz, H., 1977. Psychophysical Tests for DWI Arrest. US Department of Transportation, National Highway Traffic Safety Administration, Final Report DOT-HS-5-01242.

College of Problems of Drug Dependence, 1995. Human subject issues in drug abuse research. Drug Alcohol Depend. 37, 167-175.

Cone, E.J., Huestis, M.A., 1993. Relating blood concentrations of tetrahydrocannabinol and metabolites to pharmacologic effects and time of marijuana usage. Ther. Drug Monit. 15, 527-532.
Dai, B., 1937/1970. Opium Addiction in Chicago. Patterson Smith, Montclair, NJ.

Deehan, A., Saville, E., 2003. Calculating the risk: recreational drug use among clubbers in the South East of England. Home Office Online Report 43/03, London.

Fincham, F., Barling, J., 1979. Effects of alcohol on moral functioning in male social drinkers. J. Genet. Psychol. 134, 79-88.

Fisher, C.B., 2003. Goodness-of-fit ethic for informed consent to research involving adults with mental retardation and developmental disabilities. Ment. Retard. Dev. Disabil. Res. 9, 27-31.

Foddy, B., Savulescu, J., 2006. Addiction and autonomy: can addicted people consent to the prescription of their drug of addiction? Bioethics 20, $1-15$.

Gourley, M., 2004. A subcultural study of recreational ecstasy use. J. Sociol. 40, 59-73.

Kelly, B.C., 2006. Concepts of risk in the lives of ecstasy-using youth. In: Sanders, B. (Ed.), Drugs, Clubs and Young People. Ashgate, Aldershot, pp. 50-65.

Krober, H.-L., 1998. Psychiatric criteria of legal responsibility after the consumption of alcohol: the German situation. Eur. Addict. Res. 4, 107-112.

Lane, S.D., Cherek, D.R., Pietras, C.J., Tcheremissine, O.V., 2004. Alcohol effects on human risk taking. Psychopharmacology (Berl) 172, 6877.

Lawton, J., 2001. Gaining and maintaining informed consent: ethical concerns raised in a study of dying patients. Qual. Health Res. 11, 693-705.

Lindesmith, A.R., 1947/1968. Addiction and Opiates. Adline Publishing, Chicago.

McCrady, B.S., Bux, D.A., 1999. Ethical issues in informed consent with substance abusers. J. Consult. Clin. Psychol. 67, 186-193.

Measham, F., Aldridge, J., Parker, H., 2001. Dancing on Drugs: Risk, Health and Hedonism in the British Club Scene. Free Association Books, London.

Mouzos, J., Smith, L., Hind, N., 2006. Drug Use Monitoring in Australia: 2005 Annual Report on Drug Use Among Police Detainees. Australian Institute of Criminology, Research and Public Policy Series No. 70, Canberra.

Newcombe, R., 1992. A researcher reports from the rave. Druglink, January/February, 14-16.

Papafotiou, K., Carter, J.D., Stough, C., 2005. An evaluation of the sensitivity of the Standardised Field Sobriety Tests (SFSTs) to detect impairment due to marijuana intoxication. Psychopharmacology (Berl) V180, 107114.

Parker, H., Aldridge, J., Measham, F., 1998. Illegal Leisure. The Normalisation of Adolescent Recreational Drug Use. Routledge, London.

Perham, N., Moore, S.C., Shepherd, J., Cusens, B., 2007. Identifying drunkenness in the night-time economy. Addiction 102, 377-380.

Perrone, D., 2006. New York City club kids: a contextual understanding of club drug use. In: Sanders, B. (Ed.), Drugs, Clubs and Young People. Ashgate, Aldershot, pp. 26-49.

Preble, E., Casey, J.J., 1969. Taking care of business-the heroin user's life on the street. Int. J. Addict. 4, 1-24.

Ramcharan, P., Cutcliffe, J.R., 2001. Judging the ethics of qualitative research: considering the 'ethics as process' model. Health Soc. Care Community 9 , 358-366.

Release, 1997. Release Drugs and Dance Survey: An Insight into the Culture. Release, London.

Riley, S.C.E., James, C., Gregory, D., Dingle, H., Cadger, M., 2001. Patterns of recreational drug use at dance events in Edinburgh, Scotland. Addiction 96, 1035-1047.

Rodgers, J., 1999. Trying to get it right: undertaking research involving people with learning difficulties. Disabil. Soc. 14, 421-433.

Sanders, B., 2005. In the club: ecstasy use and supply in a London nightclub. Sociology 39, 241-258.

Santtila, P., Ekholm, M., Niemi, P., 1999. The effects of alcohol on interrogative suggestibility: the role of state-anxiety and mood states as mediating factors. Leg. Criminol. Psychol. 4, 1-13.

Schuklenk, U., 2000. Protecting the vulnerable: testing times for clinical research ethics. Soc. Sci. Med. 51, 969-977.

Silber, B.Y., Papafotiou, K., Croft, R.J., Stough, C.K.K., 2005. An evaluation of the sensitivity of the standardised field sobriety tests to detect 
the presence of amphetamine. Psychopharmacology (Berl) 182, 153159.

Silverstone, D., 2006. Pub space, rave space and urban space: three different night-time economies. In: Sanders, B. (Ed.), Drugs, Clubs and Young People. Ashgate, Aldershot, pp. 141-151.

Sudore, R.L., Landefeld, C.S., Williams, B.A., Barnes, D.E., Lindquist, K., Schillinger, D., 2006. Use of a modified informed consent process among vulnerable patients: a descriptive study. J. Gen. Intern. Med. 21, 867873.

Tossmann, P., Boldt, S., Tensil, M.-D., 2001. The use of drugs within the techno party scene in European Metropolitan Cities. Eur. Addict. Res. 7, 2-23. van de Wijngaart, G.F., Braam, R., de Bruin, D., Fris, M., Maalste, N.J.M., Verbraeck, H.T., 1999. Ecstasy use at large-scale dance events in the Netherlands. J. Drug Issue 29, 679-702.

Voas, R.B., Furr-Holden, D., Lauer, E., Bright, K., Johnson, M.B., Miller, B., 2006. Portal surveys of time-out drinking locations: a tool for studying binge drinking and AOD use. Eval. Rev. 30, 44-65.

Wiles, R., Charles, V., Crow, G., Heath, S., 2006. Researching researchers: lessons for research ethics. Qual. Res. 6, 283-299.

Yacoubian, G.S., Boyle, C., Harding, C.A., Loftus, E.A., 2003. It's a rave new world: estimating the prevalence and perceived harm of ecstasy and other drug use among club rave attendees. J. Drug Educ. 33, 187-196. 